\title{
Experimental search for evidence of the three-nucleon force and a new analysis method
}

\author{
P. Thörngren Engblom*, H.O. Meyer ${ }^{\dagger}$, B. von Przewoski ${ }^{\dagger}$, J. \\ Kuroś-Żołnierczuk $^{* *}$, T.J. Whitaker ${ }^{\dagger}$, J. Doskow ${ }^{\dagger}$, B. Lorentz ${ }^{\ddagger}$, P.V. \\ Pancella $^{\S}$, R.E. Pollock ${ }^{\dagger}$, F. Rathmann ${ }^{\ddagger}$, T. Rinckel ${ }^{\dagger}$, T. Wise ${ }^{\mathbb{I}}$, H. Witałall, \\ J. Golak ${ }^{\|}$, H. Kamada ${ }^{\dagger \dagger}$, A. Nogga ${ }^{\ddagger}$ and R. Skibiński ${ }^{\|}$ \\ ${ }^{*} I S V$, Uppsala University, Box 535, S751 21 Uppsala, Sweden \\ ${ }^{\dagger}$ Indiana University Cyclotron Facility, Bloomington, Indiana 47405, USA \\ ${ }^{* *}$ Nuclear Theory Center, Indiana University, Bloomington, Indiana 47405, USA \\ ${ }^{\ddagger}$ Institut für Kernphysik, Forschungszentrum Jülich, Jülich, Germany \\ ${ }^{\S}$ Western Michigan University, Kalamazoo, Michigan 49008, USA \\ II University of Wisconsin, Madison, Wisconsin 53706, USA \\ ${ }$ M. Smoluchowski Institute of Physics, Jagiellonian University, 30-059 Kraków, Poland \\ ${ }^{\dagger \dagger}$ Kyushu Institute of Technology, 1-1 Sensucho, Tobata, Kitakyushu 804-8550, Japan \\ $\boxplus$ Department of Physics, University of Arizona, Tucson, Arizona, 85721, USA
}

\begin{abstract}
A research program with the aim of investigating the spin dependence of the threenucleon continuum in $\vec{p} \vec{d}$ collisions at intermediate energies was carried out at IUCF using the Polarized INternal Target EXperiments (PINTEX) facility. In the elastic scattering experiment at 135 and $200 \mathrm{MeV}$ proton beam energies a total of 15 independent spin observables were obtained. The breakup experiment was done with a vector and tensor polarized deuteron beam of $270 \mathrm{MeV}$ and an internal polarized hydrogen gas target. We developed a novel technique for the analysis of the breakup observables, the sampling method. The new approach takes into account acceptance and non-uniformities of detection efficiencies and is suitable for any kinematically complete experiment with three particles in the final state.
\end{abstract}

\section{Introduction}

The goal of finding experimental evidence for a three-nucleon force, and especially to map out its spin dependence has to include the major part of possible observables in order to be conclusive. A prerequisite for the interpretation of the data is the possibility to compare the results to computationally exact solutions of the three-nucleon system. To meet this theoretical challenge the Cracow-Bochum group has performed Faddeev calculations up to the pion production threshold [1]. Using modern high-precision nucleonnucleon (NN) potentials in combination with models for three-nucleon forces (3NF), parameter-free predictions for scattering observables can be obtained. Deviations from $\mathrm{NN}$ behaviour and agreement with calculations incorporating a 3NF model is thus considered to be the signature for the existence of 3NFs. Considerable experimental activity over the last few years has been aimed primarily at measuring vector and tensor analyzing powers in pd elastic scattering, recently also several transfer coefficients were reported ([2] and references therein). 


\section{The PINTEX pd elastic scattering experiment}

In a Cooler storage ring experiment a polarized proton beam at 135 and $200 \mathrm{MeV}$ was used in conjunction with a vector and tensor polarized deuterium gas target. The advanced options of the facility to control and change the spin alignment axis of both protons and deuterons allowed all analyzing powers and 10 of 12 possible spin correlation coefficients to be measured [3]. The results at $135 \mathrm{MeV}$ are shown in Fig 1. The agreement of the data with NN potential predictions is fairly good. The effects of the inclusion of a $3 \mathrm{NF}$ was studied and did not improve the agreement between theory and data in any consistent way with respect to scattering angle, energy or particular $3 \mathrm{NF}$ model (see figures 10-12 of ref [3] )

\section{The dp breakup channel}

The analysis of the dp breakup observables has been somewhat restricted by the fact that comparison to theory could only be done over limited phase space regions as a function of the kinematically allowed locus in the plane of the energies of the two detected nucleons (the so called S-curve). In order to overcome this deficiency and make use of the relatively large acceptance of the PINTEX facility we developed a new analysis technique; the sampling method [4].

For a three-particle final state to be kinematically fully determined, five parameters are required. When extracting observables one has to choose which independent variable to use and what regions of phase space to integrate over. The acceptance and any significant efficiency variation has to be well known, most often this is accomplished by advanced monte carlo simulations. However, with the complete kinematical information of an event as input, the theoretical prediction for the sought observable can be calculated for that particular event. A given experimental data set $(\gamma)$, containing the phase space points $\left(x_{i}\right)$ used as input to a theoretical model calculation, can then be compared directly to theory by taking the mean of the calculated theoretical predicted values: $O^{t h}(\gamma)=\frac{\sum O^{t h}\left(x_{i}\right)}{N(\gamma)}=<O^{t h}>$, where $N(\gamma)$ is the total number of events. The statistical error of the theoretical prediction that appear from the randomness of the sampling, is given by the standard deviation. In case the theoretical computations are very time consuming, as when the Faddeev amplitudes are needed, we adopt multidimensional linear interpolation on a grid of precalculated stored theoretical values, for details see [4]. The sampling method using a grid is particularly useful in planning experiments and in governing a complicated data analysis. See Fig. 2 for examples of theoretical predictions for the tensor analyzing powers in dp breakup as measured by our experimental setup.

For the experiment the deuteron beam was prepared in five different polarization states, unpolarized, two pure tensor $( \pm)$, and two mixed vector $( \pm)$ and tensor + states. The target was a thin $(25 \mu \mathrm{m})$ Teflon-coated aluminum storage cell filled by an atomic beam of polarized hydrogen atoms. The four-momenta of the two outgoing protons were measured in a forward detector stack subtending a polar angle of 45 degrees [5].

The sampling method was applied to the analysis of axial observables in breakup [6]. The experiment was motivated by the prediction that axial observables may exhibit 


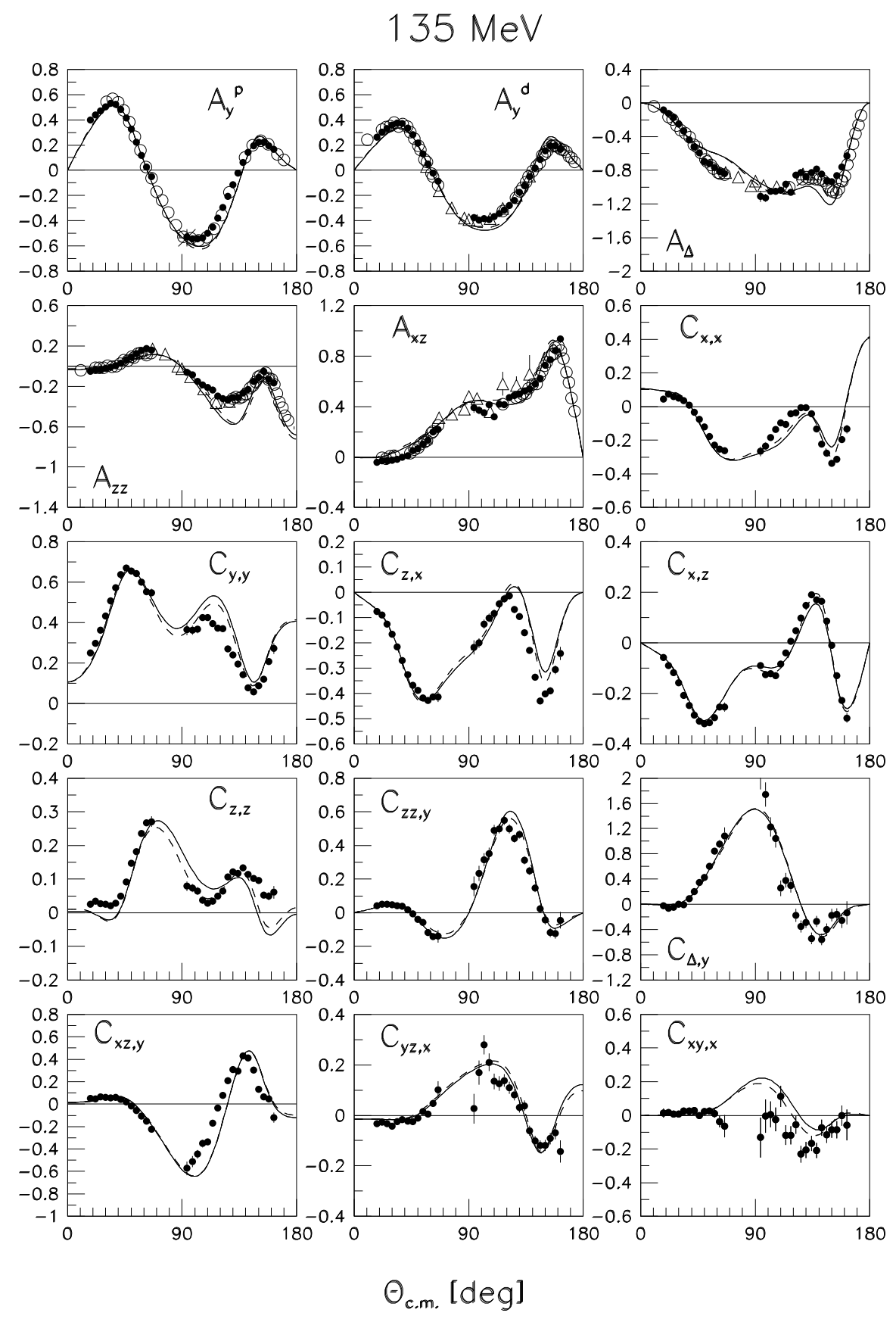

FIGURE 1. Spin observables at $135 \mathrm{MeV}$ from the present experiment (full circles) together with previously existing data (empty markers) and theoretical predictions from NN potentials: CDBonn (full line) [7], AV18 [8](dashed line). Figure taken from [3].

enhanced sensitivity to the $3 \mathrm{NF}$ due to a particular kind of spin operators present in a $3 \mathrm{NF}$ but absent in NN interactions [9]. Axial observables vanish for coplanar events since parity is conserved, whereas they may be non-zero in any other configuration of a breakup reaction. It was found that the agreement with theory was not improved by including a $3 \mathrm{NF}$ in the calculations and thus the expectations concerning axial observables was not validated by the experiment. 

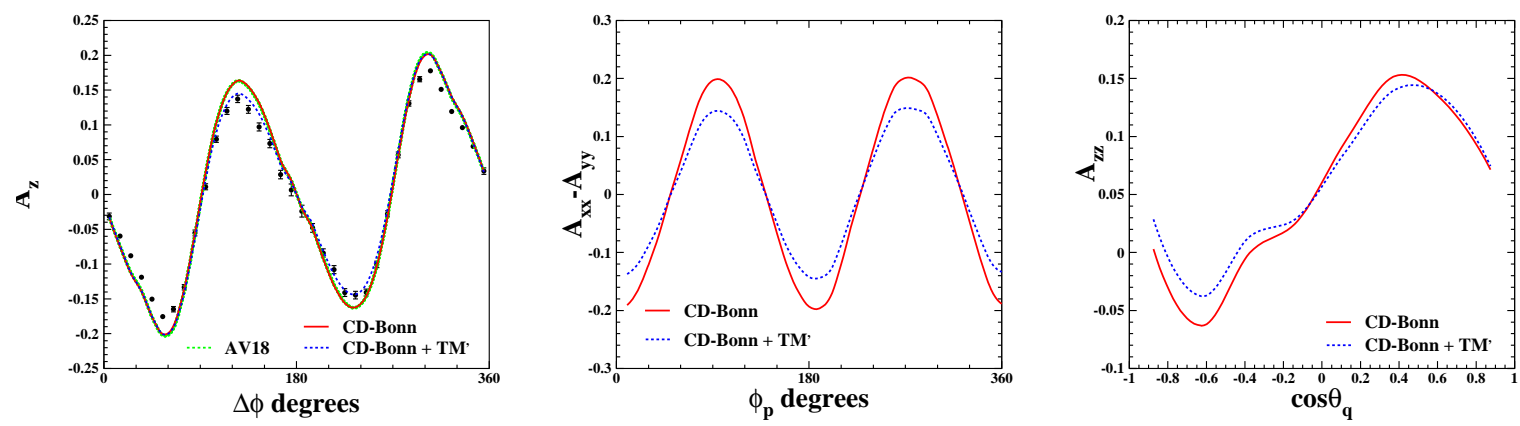

FIGURE 2. Left: Measured $A_{z}$ as a function of $\Delta \phi=\phi_{p}-\phi_{q}$, (where $p$ and $q$ are the jacobi momenta in the $\mathrm{cm}$ ), compared to NN and 3NF calculations. Middle \& Right: Predicted 3NF sensitivity for $A_{x x}-A_{y y}$ and $A_{z z}$ for the present experimental setup obtained by the sampling method and interpolation. As input phase space coordinates for the interpolation were used: a data sample (middle panel) and phase space distributed montecarlo generated events (right panel).

\section{Summary}

Large values of axial observables in $\vec{d} \vec{p}$ breakup were reported for the first time using a new analysis technique, applicable to any three-particle final state that is fully determined. The analysis of the breakup tensor analyzing powers is in progress with the objective to compare with theoretical predictions extracted from an NN potential at fourth order of ChPT [10]. The currently available 3NF models are not confirmed by our measurements of pd elastic and breakup reactions.

\section{ACKNOWLEDGMENTS}

The authors wish to thank the IUCF Operations Group for their enthusiastic work at all hours. The work has been done under NFS Grant No. PHY-0100348, DOE Grant No. FG0288ER40438 and Grant No. Dnr. 629-2001-3868 from the Swedish Research Council.

\section{REFERENCES}

1. H. Witala et al., Phys. Rev. C63, 024007 (2001)

2. K. Sekiguchi et al., in these proceedings

3. B. von Przewoski et al., acc. for publ. Phys. Rev. C

4. J. Kuroś-Żołnierczuk, P. Thörngren Engblom, H-O. Meyer, T. J. Whitaker, H. Witała, J. Golak, H. Kamada, A. Nogga, R. Skibiński, Few-Body Syst. 34, 259-273 (2004) |arXiv:nucl-th/0402030|

5. T. Rinckel et al., Nucl. Inst. Meth. A43, 117 (2000)

6. H.O. Meyer et al., Phys. Rev. Lett. 93, nr 11 (2004), T.J. Whitaker, PhD thesis, IUCF (2004)

7. R. Machleidt, Phys. Rev. C63, 024001 (2001)

8. R.B. Wiringa, V.G. Stoks and R. Schiavilla, Phys. Rev. C51, 38 (1995)

9. L.D. Knutson, Phys. Rev. Lett.73, 3062 (1994)

10. D.R. Entem and R. Machleidt, Phys. Rev. C 68, 041001 (2003) 